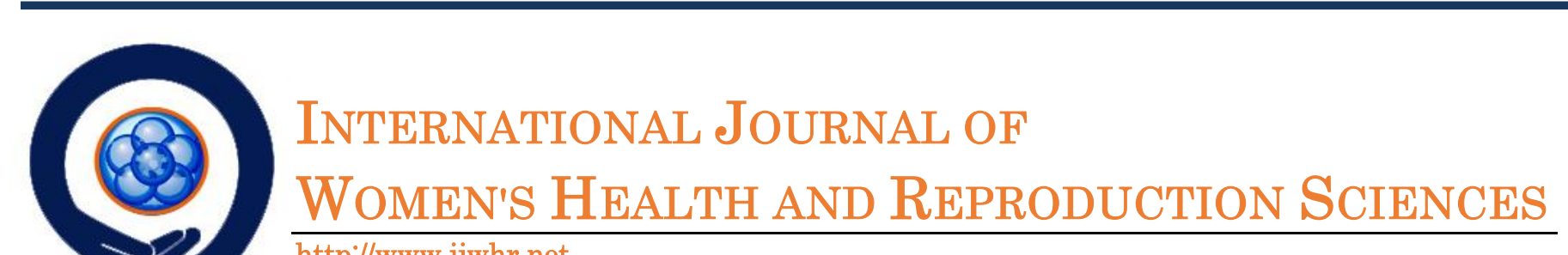

\title{
Prediction of Age at Menopause in Women of Suburban Areas in Chennai Using A Model of Fsh Over Age - A Pilot Study
}

\author{
SM Priyadharshini ${ }^{1}$, VS Kalaiselvi ${ }^{2 *}$, K Prabhu ${ }^{3 *}$, P Devaraj ${ }^{4}$, Prakash S ${ }^{5}$, \\ K Prasanth ${ }^{6}, V^{2}$ Prasanna E ${ }^{7}$
}

\section{Article History:}

Received 6 December 2013

Accepted 3 February 2014

Revised 19 January 2014

Available online 4 February 2014

\section{Keywords:}

Amenorrhea

Anti-Mullerian Hormone

Eumenorrhea

Follicle-Stimulating Hormone

Menopause

Oligomenorrhea

\section{Corresponding Author:}

K Prabhu, Department of Anatomy, Sree Balaji Medical college \& Hospital, Chennai, Tamil Nadu, India.

Tel: +919841171185

Email: prb_anu@yahoo.co.in

\begin{abstract}
Objectives: The human ovary is characterized by early senescence and the end stage of ovarian activity is termed menopause. The age at which menopause occur is between 45 and 55 years world wide. The objective of this pilot study is to determine the age at menopause by using a model of FSH over age in women of sub urban region around Chennai, India.

Materials and Methods: The subjects include 500 patients of age between 30 and 36 yrs with BMI ranging from 24-28.After recording their general profile and history, blood samples were obtained by venipuncture and hormone FSH was estimated on the day 3 of the menstrual cycle. Based on functional dependence of FSH in the form of exponential relation with age, a model was proposed. Using least square approximation the beta values were calculated.

Results: With the help of beta values and using the cut off value of $40 \mathrm{IU} / \mathrm{ml}$ for FSH, this predicted model determined the age of menopause as 44.6yrs in women of sub urban region around Chennai.

Conclusion: The age of menopause is different in various region worlds wide. According to this pilot study the suburban women of Chennai, attain menopause at an age of 44.6 years. Further exploration should be done to alleviate the role of diet, life style and ethnic variation on menopausal age and the impact of chronic disease like osteoporosis during the period of menopause.
\end{abstract}

1 - Department of Gynecology \& Obstetrics, Sree Balaji Medical College \& hospital, Chennai, Tamil Nadu, India.

2- Department of Biochemistry, Sree Balaji Medical College \& hospital, Chennai, Tamil Nadu, India.

3- Department of Anatomy, Sree Balaji Medical college \& Hospital, Chennai, Tamil Nadu, India.

4- Department of Mathematics, Anna University, Chennai, Tamil Nadu, India.

5- Department of Anatomy, Dr.ALMPGIBMS, University of Madras, Taramani, Chennai, Tamil Nadu, India.

6- Sri Ramachandra Medical College and Hospital, Chennai, Tamil Nadu. India.

7- Sree Balaji Medical College \& hospital, Chennai, Tamil Nadu, India. 


\section{Introduction:}

The human ovary contains a fixed pool of primordial follicles which is called ovarian reserve. The ovarian reserve is maximum around 5th months of gestational age and culminating in menopause at an average age of 50-51 yrs. The size of the follicle pool and its rate of decline can be estimated at any age using the mathematical model by Faddy and Gosden (1). Reproductive aging in women is based on the depletion of ovarian follicles with 1000 follicles remaining at the menopause (2).This progressive reduction in ovarian reserve is attributed to follicle death by apoptosis. Follicle depletion as a result of atresia and recruitment towards ovulation leads to premature exhaustion of the follicle pool and menopause long before death (3).

There are no clinical markers to assess the exact ovarian reserve. The number of antral follicles and the total ovarian volume measured by trans vaginal ultrasound $(4,5,6)$,basal FSH $(7)$ Inhibin B ( 8), Estradiol (9) and Inhibin response to exogenous GnRh agonist (GAST) (10) or FSH stimulation (EFFORT) (11) have been mentioned in the literatures to predict declining fertility related to reproductive aging.

The progressive decline in the quality and quantity of oocytes in primordial, intermediate and primary follicle is attributed to reproductive aging. The elevated level of FSH and estradiol in the early follicular phase and shortened menstrual cycle were observed to be associated with diminished ovarian reserve. The other hormone which is associated with prediction of menopause is anti-mullerian hormone (AMH). The word menopause which was used first time by French physician De Gardanne, is characterized endocrinologically by evidence of decreasing ovarian activity, biologically by decreasing fertility and clinically by alternation in menstrual cycle intervals. Naturally menopause is recognized to have occurred after 12 consecutive months of amenorrohea for which there is no other obvious pathological or physiological cause (WHO report). The age at which menopause occurs is between 45 and 55 years for women world wide. In developed countries the average age of menopause is about 51 years (12-15) whereas in countries like Philippines, and in various parts of Africa, India, Pakistan and Thailand it is reported to be $45-50$ yrs (16-23).

Flint (24) was one of the first to carry out community based investigation on menopause among Indian women. Very little research has been done on menopause in Indian context as there is a need to recognize menopause as an issue in women health care. In this pilot study a predicted model of FSH over age in the reproductive period was proposed to determine the occurrence of menopause in local population in Chennai.

\section{Material \& Methods:}

The present work was approved by the institutional ethical committee of Sree Balaji medical college and informed consent was obtained from all the patients. . Five hundred female subjects with age ranging from 30$36 y r$ and BMI ranging from 24-31 were included in this study They were recruited from Prashanth Fertility Centre, Chennai, Tamil nadu, India from 2009 to 2011 including patients with history of oligomenorrhea (interval between periods $>35$ days) amenorrhea (absence of vaginal bleeding for at least six months) and eumenorrhea (normal menstrual cycle). Serum FSH concentrations are within normal limits (1-10 IU/L). The subjects with endocrine disorders or any other related disease either thyroid problems or diabetes, use of medications or oral contraceptives during last three months were excluded in this study. Blood samples were obtained by venipuncture and processed within $2 \mathrm{hrs}$ after withdrawal and serum was separated and stored at $-20{ }^{\circ} \mathrm{C}$ until assayed. FSH was estimated on day 3 after the last menstrual period by ELISA (FSH (MONOBIND Inc).

\section{Statistical Analysis :}

Association between FSH and age, and its changes over time were assessed. Based on above functional dependence of FSH in the form of exponential relation with age i.e. A MODEL AGE $=\beta 0(\mathrm{FSH}) \beta 1$ using least square approximation was proposed. This model will explain how least square approximation can be used to predict the 
menopause, which is the end stage of ovarian aging. The value of $40 \mathrm{mIU} / \mathrm{mL}$ for FSH was used as cutoff value to predict menopause. The age at which the subjects under study reach menopause were found using the above least square fit model.

\section{Results:}

The mean age of the subjects and BMI involved in this study were 33.4 yrs and 27.5 yrs respectively. In this predicted model of FSH over age the value of $\beta 0$ and $\beta 1$ were 41.4 and 0.17 respectively. As we substitute the value of $40 \mathrm{Iu} / \mathrm{ml}$ for FSH in this model to predict menopause, it was found to be at 44.6 yrs in the sub-urban population of Chennai . The positive correlation between FSH and age in the early and late transition period of menopause was seen .

\section{Discussion:}

The mean age of menopause in the present study is similar to those reported for rural women in north India (44.1 yrs) (25) and Baroda females 44.59yrs (26). While comparing certain international studies, we found similar results in Pakistan women (47.42yrs) and for Turkish women (44 yrs) (27). But Japanese women with 49.33 yrs (13), Iranian women with 49.6 yrs (15) and American women with 50.9 yrs (28) had higher mean age of menopause than the current study.

The number of menopausal women's are projected to increase rapidly from a total of 467 million to 1200 million by 2030 all around the world. The number of menopausal will rise substantially faster in the developing countries than in industrialized world. The proportion of menopausal women in the total population will increase from $9 \%$ in 1990 to $14 \%$ in 2030 (29).The total of 130 million Indian women are expected to live beyond menopause into old age by 2015 .

The reproductive aging in women progresses through three stages namely reproduction, the menopausal transition and finally post menopause. Before reaching menopause there are various stages like pre menopausal, early menopausal transition and late menopausal transition. No reliable markers are available to differentiate each stage of reproductive stage. AMH appear to differentiate the early premenopausal stage to late menopausal transition and however level appear to drop below detection during the transition and less useful in determining late transition from menopause onset (30). FSH and E2 in the early follicular phase of the menstrual cycle reflects the sum of both hypothalamic drive and ovarian feed back but is not elevated until the peri-menopausal stage. The follicular phase of the menstrual cycle in older women becomes considerably shorter and produces higher E2 levels in older women when compared with younger women. So rise in the follicular phase of the estradiol and FSH levels may be observed during the late menopausal transition (31) and so its level can be detected easily to predict menopause. In premenopausal stage the FSH level will be less than $10 \mathrm{mIU} / \mathrm{mL}$. The early menopausal transition begins approximately 7- 8 years prior to the final menopausal period. It involves shortening of the menstrual cycles and FSH values are generally greater than $10 \mathrm{mIU} / \mathrm{mL}(31,32)$. The late menopausal transition begins 3 to 4 years prior to final menopause period and the women experiences 60 or more days of amenorrhea and FSH values are greater than 30 to $40 \mathrm{IU} / \mathrm{mL}$ (31). Most studies show that FSH rises from the early menopausal transition to post menopause $(31,32)$, but subtle changes may be observed earlier between the late reproductive stage and the early menopausal transition. Sowers et al (33) showed that FSH will rise modestly before 7 years prior to the final menopausal period (FMP), with a major acceleration in rise between 7 and 2 years prior to the FMP and acute increase in rise between 2 years prior and 1 year following FMP. As FSH has more advantages to predict menopause rather than other reliable markers, in this study using the model of FSH over age was proposed and predicted the age of menopause to be 44.6 yrs in women of suburban areas in Chennai.

India is a large country with a wide variety of environmental conditions. It shows ethnic multiplicity and is characterized by an interracial mixing rarely seen in other countries. Taking into account of these factors the data base obtained in our study 
may not be representative of the entire Indian population and therefore our normative data should be used only for a population sharing the same genetic potential and living under similar environmental conditions.

Generally the median age at menopause is fifty one. Approximately $1 \%$ of women will reach menopause by age of $40,10 \%$ by age of 46 and $90 \%$ by age of 55 (15). This study alleviates that predicted model of FSH over age determine menopause to occur at 44.6yrs.India with a wide ethnic variation has a population of more than 100 cores and undergone significant economic and cultural changes over the past two decades. Changes in life style reflect their diet and physical activity. Flint (24) was one of the first to carry out a community based investigation on menopause among women and very little research has been done on menopause in Indian context as there is a need to recognize menopause as an important issue in health case of women.

\section{Conclusion :}

According to this pilot study using the model of FSH over age, the menopausal age was found to be 44.6 yrs. The prediction of early and late menopause in this population help to identify onset of several chronic diseases like osteoporosis, cardio vascular diseases and certain carcinomas like breast associated with it, that can be treated at the earliest. As very little research has been done on menopause in Indian context, there is a need to recognize menopause as an important issue in women health care. Further exploration should be done to alleviate the role of diet, life style and ethnic variation on menopausal age.

\section{Conflicts of interest:}

Authors declare that there is no any conflict of interest.

\section{Acknowledgments:}

We thank Sree Balaji Medical College \& hospital, Chennai, Tamil Nadu India and Prashanth Fertility Centre, Chennai, Tamil nadu, India for their support during this work.

\section{References:}

1. Faddy MJ and Gosden RG. A model conforming the decline in follicle numbers to the age of menopause in women Hum Reprod.1996; 11: 1484-1486.

2. Faddy MJ, Gosden RG, Gougeon A, Richardson SJ, and Nelson JF. Accelerated disappearance of ovarian follicles in mid life; implications for forecasting menopause. Hum Reprod.1992; 7: 1342-1346.

3. Te velde ER, and Pearson, PL.The variability of female reproduction ageing. Hum. Reprod update. 2002; 8:141-154.

4. Lass, A., Skull, J, McVeigh, E, Margara, R. and Winston, R.M. Measurement of ovarian volume by transvaginal sonography before ovulation induction with human menopausal gonadotrophin for in-vitro fertilization can predict poor response. Hum. Reprod.1997; 12: 294297.

5. Chang MY, Chiang CH, Hsieh TT, Soong Yk, Hsu KH. Use of the antral follicle count to predict the outcome of assisted reproductive technologies. Fertil Steril. 1998;69:505-510 
6. Bancsi LF, Broekmans FJ, Eijkemans MJ, de Jong FH,Habbema JD, te Velde ER. Predictors of poor ovarian response in in vitro fertilization: A prospective study comparing basal markers of ovarian reserve. Fertil Steril. 2002;77:328-336

7. Bancsi LF, Huijs AM, den Ouden CT, Broekmans FJ, Looman CW, Blankenstein MA, te Velde ER. Basal folliclestimulating hormone levels are of limited value in predicting ongoing pregnancy rates after in vitro fertilization. Fertil Steril. 2000;73:552-557

8. Creus M, Penarrubia J,Fabregues F, Vidal E, carmona F, Casamitjana R, Vanrell J A and Balasch J.Day 3 serum inhibin B and FSH and age as predictors of assisted reproduction treatment outcome. Hum . Reprod . 2000 ;15: 2341-2346.

9. Evers. J.I Slaats, P.. Land, J.A., Dumoulin, J.C and Dunselman, G A 8) Elevated levels of basal estradiol-17beta predict poor response in padfnts with normal basal levels of folliclestimulating hormone undergoing in vitro fertilization. Fertil. Steril.1998; 69: 1010-1014.

10. Avrech OM, Royburt M, Sabah G, Zukerman Z, Pinkas H,Amit S, Ovadia J, Fisch B.The initial flare-up induced by gonadotropin releasing hormone agonist may serve as a predictor of ovarian response in the current IVF-ET treatment cycle in normogonadotropic women aged 40-48 years. J Assist Reprod Genet. 1996;13:395-400

11. Fanchin R, de Ziegler D, Olivennes F, Taieb J, Dzik A, Frydman R: Exogenous follicle stimulating hormone ovarian reserve test (EFORT): A simple and reliable screening test for detecting 'poor responders' in in-vitro fertilization. Hum Reprod. 1994;9:1607-1611

12. Suzzanne A. Llewellyn-Jones D, Perz P. Changes in Australian women's perception of the menopause and menopausal symptoms before and after the climacteric. Maturitas. 1995; 20: 121-128.

13. Kazem M, Hashemi SMS, Farahaui FKA, Age at natural menopause in Iran. Maturitas. 2004; 49: 321-6.

14. Sukwauma P, Meekliangvan J, 'I'amrongterakul T, Tanapat Y. Savarait SA, Bootijittpimoii P. Menopausal symptoms among Thai women in Bangkok, Maturitas. 1991; 13:217-8.

15. Mckinlay SM. Brambilla DJ, Posner JS. The normal menopause transition. Maturitas. 1992; 4: 37-46.

16. Brambilla DJ. Mckinlay SM. A prospecting study of factors affecting age at menopause. J Clin Epidemiol 1989; 42(11); 103 I -9.

17. Richardson SJ. The biological Basis of Menopause. Bailier's Clinic. Endocrinol Metab 1993:7: 1-16.

18. Goodman MJ, Sstioke-Griffin A. Griffin P. Grove J. Menarche, pregnancy, birth spacing and menopause among the Agta women for ages of Cagayan Province: Luzon the Phillipines Ann Hum Biol. 1985; 12: 169-77. 
19. Randhawa I, Premi HK, Gupta T. The age at menopause ill the women of Himachal Pradesh, and the factors affecting the menopause. Ind J Public Health 1987; 21(1): 40-4.

20. Talukdat S. On age at menopause. Man India. 1978; 54(4): 345-50.

21. Wasti R. Robinson SC, Khan Y. Bavamddin N. Characteristics of menopause in three groups in Karachi. Pakistan. Maturitas. 1993; 16: 61-9.

22. Tanner JM. Growth at adolescence. Oxford: Blackwell Scientific Publication 1962.

23. Laslett P. Age at menarche in Europe since the eighteenth century. Int Discip Hist. 1977; 2: 221-36.

24. Flint; M. Menarehe and menopause of Rajput women. Ph.D dissertation, New York: University of New York 1974.

25. Singh A, Arora AK. Profile of menopausal women in rural north India. Climateric. 2005 : 8(2): $177-84$.

26. Nagar S, Dave P,. Perception of women towards physiological problems faced at menopause . Anthropologist. 2005;7(3):173-5.

27. Ozdemier O. col M. The age at menopause and associated factors at the health centre in Ankara Turkey Maturitas 2004;49:211-9.

28. Hagstad A.Gynecology and sexuality in middle aged women. Women's health 1988;13:5780 .

29. Hill K- 'The demography of menopause. Maturitas. 1996; 23: I 13 $\neg 27$.

30. Knauff EA, Eijkemans MJ, Lambalk CB, et al. AntiMullerian hormone, inhibin B, and antral follicle count in young women with ovarian failure. J Clin Endocrinol Metab. 2009;94(3):786-792.

31. Burger HG, Dudley EC, Hopper JL, et al. The endocrinology of the menopausal transition: a crosssectional study of a population-based sample. J Clin Endocrinol Metab. 1995;80(12):3537-3545.

32. Gracia CR, Sammel MD, Freeman EW, et al. Defining menopause status: creation of a new definition to identify the early changes of the menopausal transition. Menopause. 2005;12(2):128-135.

33. Sowers MR, Zheng H, Mc Connell D, et al. Follicle stimulating hormone and its rate of change in defining menopause transition stages. J Clin Endo Crinol Metab. 2008; 93(10);39583964. 